\title{
Operational Modal Analysis Based on Subspace Algorithm with an Improved Stabilization Diagram Method
}

\author{
Shiqiang Qin, Juntao Kang, and Qiuping Wang \\ School of Civil Engineering and Architecture, Wuhan University of Technology, Wuhan 430070, China \\ Correspondence should be addressed to Juntao Kang; jtkang@163.com
}

Received 23 June 2015; Revised 2 November 2015; Accepted 3 November 2015

Academic Editor: Mickaël Lallart

Copyright (C) 2016 Shiqiang Qin et al. This is an open access article distributed under the Creative Commons Attribution License, which permits unrestricted use, distribution, and reproduction in any medium, provided the original work is properly cited.

\begin{abstract}
Subspace-based algorithms for operational modal analysis have been extensively studied in the past decades. In the postprocessing of subspace-based algorithms, the stabilization diagram is often used to determine modal parameters. In this paper, an improved stabilization diagram is proposed for stochastic subspace identification. Specifically, first, a model order selection method based on singular entropy theory is proposed. The singular entropy increment is calculated from nonzero singular values of the output covariance matrix. The corresponding model order can be selected when the variation of singular entropy increment approaches to zero. Then, the stabilization diagram with confidence intervals which is established using the uncertainty of modal parameter is presented. Finally, a simulation example of a four-story structure and a full-scale cable-stayed footbridge application is employed to illustrate the improved stabilization diagram method. The study demonstrates that the model order can be reasonably determined by the proposed method. The stabilization diagram with confidence intervals can effectively remove the spurious modes.
\end{abstract}

\section{Introduction}

In recent years, the operational modal analysis (OMA) of large-scale civil structures is a continuing research topic. Compared to conventional input-output modal analysis, OMA has several advantages. First, ambient excitations are freely accessible, meaning that special excitation devices are not necessary. Then, the operational service is not interrupted during the ambient vibration test. In fact, ambient excitation is the only practical way to excite large-scale civil structures [1]. An extensive review of OMA and its application in civil engineering has been summarized by Rainieri and Fabbrocino [2]. The experimentally determined modal parameters, including natural frequency, damping ratios, and mode shapes, often can be used to update preliminary finite element models [3-6], detect structural damages [7, 8], and assess health condition of structures $[9,10]$. Furthermore, it is reported that robust OMA methods can monitor the dynamic properties of both civil [11,12] and geotechnical structures [13] before and after earthquake, thus providing strategies for rapid postearthquake emergency management. Not only is the operational safety assured, but also the cost for maintenance and management is reduced by vibration-based (modal-based) structural damage identification and assessment techniques. Therefore, accurately identifying modal parameters of civil structures from output-only response is meaningful.

Stochastic subspace identification (SSI) is a robust and efficient modal identification algorithm in time domain among many system identification techniques. At present, two implementations of SSI can be found: data driven SSI (SSI-data) and covariance driven SSI (SSI-cov). The main difference is that SSI-data directly deals with raw output data while SSI-cov works on the output correlations. Similar results can be obtained from SSI-data and SSI-cov, but SSIcov provides an easy way to estimate the uncertainties of modal parameters. Successful applications of SSI in system identification of civil structures can be found in existing literatures (see, for instance, [14-18]). Although the robustness and effectiveness of SSI for OMA have been extensively demonstrated, it is still worth improving the accuracy of the extracted modal parameters and reducing the computational cost of the algorithm. Rainieri et al. [19] proposed a procedure for accurate damping estimation under operational 
conditions by SSI. Their study gave a contribution towards standardization of testing and data processing procedures to make the error bounds of modal damping ratio estimates as low as possible. Reynders et al. [20] introduced modal transfer norm in stabilization diagram so that the physical and mathematical modes can be removed. Döhler et al. [21] proposed a memory efficient algorithm for uncertainty quantification of modal parameters estimated from multisetup SSI. As for the computational time, Rainieri [22] investigated the effects of analysis parameters on the computational time and the accuracy of modal parameter estimates, providing some useful hints for reduction of computational time and control of accuracy of estimates.

In OMA, there are mainly three steps in the SSI algorithm: (1) data preprocessing; (2) system identification; and (3) postprocessing. The postprocessing step is also called modal analysis, which is to select modal parameters from stabilization diagram (SD). At present, SD has become a standard tool in the SSI algorithm. Existing literature on SD for postprocessing of subspace identification covers mainly two subjects: the elimination of spurious modes in SD (see, for instance, $[20,23])$ and the discussion of fully automated modal analysis (see, for instance, [24-26]). However, the model order selection and the spurious modes elimination using error bounds of modal parameter estimates are less well investigated. Some relevant studies in the literature are summarized as follows. The theory of SSI algorithm (see, for instance, [18]) showed that the model order is equal to the number of nonzero singular values of block Toeplitz matrix. However, the singular values are all different from zero due to the presence of noises. Peeters and De Roeck [15] introduced SD for determining model order, while model order is usually overspecified by simply choosing a large number. Rainieri and Fabbrocino [27] investigated the influence of model order and number of block rows on the accuracy of modal parameters. Their study demonstrated that more accurate modal parameter estimates can be obtained by appropriate selection of model order and number of block rows. Different form the above mentioned methods, this paper focuses on model order selection and spurious modes elimination by presenting an improved SD method. The proposed model order selection method mainly based on singular entropy theory and the spurious modes in $\mathrm{SD}$ can be removed by introducing confidence intervals of modal parameter estimates.

The context of this paper is organized as follows. In Section 2.1, the theory formulation of SSI-cov is briefly introduced. The model order selection method based on singular entropy theory and SD with confidence intervals is described in Sections 2.2 and 2.3, respectively. To illustrate the improved SD method, a simulation example of a four-story structure is given in Section 3. The improved SD is applied to the OMA of a full-scale footbridge in Section 4, before finishing with some concluding remarks in Section 5.

\section{Theory Formulations}

2.1. SSI-cov Algorithm. The stochastic state-space model can be described by

$$
x_{k+1}=A x_{k}+w_{k}
$$

$$
y_{k}=C x_{k}+v_{k}
$$

where $x_{k}$ is the state vector; $y_{k}$ is output vector; $A$ and $C$ are the state matrix and output matrix, respectively; $w_{k}$ and $v_{k}$ stand for the modelling errors and test inaccuracies. Their covariance matrices are defined as

$$
\mathrm{E}\left[\left(\begin{array}{c}
w_{p} \\
v_{p}
\end{array}\right)\left(\begin{array}{ll}
w_{q}^{T} & v_{q}^{T}
\end{array}\right)\right]=\left(\begin{array}{cc}
Q & S \\
S^{T} & R
\end{array}\right) \delta_{p q},
$$

where E denotes mathematical expectation and $\delta_{p q}$ is the Kronecker operator. The main idea of SSI-cov is to identify system matrices, including state matrix $A$ and output matrix $C$. The modal parameters can be extracted by eigenvalue decomposition of $A$. To realize the idea, the algorithm starts from computing the output covariance $R_{i}$ defined by

$$
R_{i}=\mathrm{E}\left[\begin{array}{ll}
y_{k+i} & y_{k}^{T}
\end{array}\right]=\lim _{N \rightarrow \infty} \frac{1}{N} \sum_{k=0}^{N-i} y_{k+i} y_{k}^{T}
$$

where $N$ is the data samples. With (3), the output covariance matrix $T_{1 \mid i}$ (also named Toeplitz Matrix) can be obtained from

$$
T_{1 \mid i}=\left(\begin{array}{cccc}
R_{i} & R_{i-1} & \cdots & R_{1} \\
R_{i+1} & R_{i} & \cdots & R_{2} \\
\vdots & \vdots & \ddots & \vdots \\
R_{2 i-1} & R_{2 i-2} & \cdots & R_{i}
\end{array}\right)
$$

The Toeplitz matrix $T_{1 \mid i}$ has dimensions $l i \times l i$, where $l$ is the number of outputs. In (4), $R_{i}$ is an unbiased estimate of the true correlation matrix at time lag $i$ based on finite number of data samples. The value of $i$ is usually determined so that $l i \geq n$, where $n$ is the model order [27]. This means that the time lag parameter $i$ can be determined when model order $n$ is selected.

According to the property of the stochastic state-space model, the Toeplitz matrix can be decomposed into the product of observability matrix $O_{i}$ and controllability matrix $\Gamma_{i}$ :

$$
T_{1 \mid i}=\left(\begin{array}{c}
C \\
C A \\
\vdots \\
C A^{i-1}
\end{array}\right)\left(\begin{array}{llll}
A^{i-1} G & A^{i-2} G & \cdots & G
\end{array}\right)=O_{i} \Gamma_{i} .
$$
yields

Meanwhile, the singular value decomposition of $T_{1 \mid i}$

$$
\begin{aligned}
T_{1 \mid i} & =U S V^{T}=\left(\begin{array}{ll}
U_{1} & U_{2}
\end{array}\right)\left(\begin{array}{cc}
S_{1} & 0 \\
0 & S_{2}=0
\end{array}\right)\left(\begin{array}{c}
V_{1}^{T} \\
V_{2}^{T}
\end{array}\right) \\
& =U_{1} S_{1} V_{1}^{T},
\end{aligned}
$$


where $U$ and $V$ are the orthogonal matrices and $S$ is the diagonal matrix with singular values. The system matrices $(A, C)$ can be solved by simultaneously solving (5) and (6).

To obtain the modal parameters, the eigenvalue decomposition is applied to the state matrix $A$ :

$$
A=\Psi \Lambda \Psi^{-1}=\sum_{k=1}^{n} \psi_{k} \lambda_{k} \psi_{k}
$$

In (7), $\Lambda=\operatorname{diag}\left(\lambda_{i}\right), i=1,2, \ldots, n$, are eigenvalues of $A ; \lambda_{k}$ is the eigenvalue of discrete time system; $\Psi$ is the eigenvector of $A$.

The natural frequency $f_{k}$, damping ratio $\xi_{k}$, and mode shape $\phi_{k}$ are obtained from

$$
\begin{aligned}
f_{k} & =\frac{\left|\lambda_{c k}\right|}{2 \pi}, \\
\xi_{k} & =\frac{-100 \operatorname{Re}\left(\lambda_{c k}\right)}{\left|\lambda_{c k}\right|}, \\
\phi_{k} & =C \psi_{k},
\end{aligned}
$$

where $\lambda_{c k}$ is the eigenvalue in continuous time system, obtaining from

$$
\lambda_{c k}=\frac{\ln \lambda_{k}}{\Delta T}
$$

Since SSI-cov is a well-known algorithm, the detailed theory is not introduced in this paper. The readers could refer to [14].

2.2. Model Order Selection. One parameter which needs to be determined in SSI-cov algorithm is the model order $n$. Since the modal parameters existed in the form of conjunction pairs, the model order should be an even number. Theoretically, the model order equals to the number of nonzero singular values of Toeplitz matrix. However, the singular values converge to zero little by little in practice due to the influence of noise. It is very difficult to determine $n$ by observing the singular values-model order plot. Thus, the SD method is introduced. By selecting a comparatively large number $n_{\text {max }}$ for model order, the modal parameters are solved from $2: 2: n_{\max }$. Those calculated modal parameters satisfying stable criterion are called stable points and shown in frequencymodel order plot. The commonly used stable criterions are

$$
\begin{aligned}
& \Delta f_{i}=\frac{f_{i+1}-f_{i}}{f_{i}} \times 100 \% \leq 1 \%, \\
& \Delta \xi_{i}=\frac{\xi_{i+1}-\xi_{i}}{\xi_{i}} \times 100 \% \leq 5 \%, \\
& \left(1-\operatorname{MAC}\left(\phi_{i}, \phi_{i+1}\right)\right) \times 100 \% \leq 2 \%,
\end{aligned}
$$

where MAC is the modal assurance criterion of mode shapes.

Although the stabilization diagram has been well recognized, it is still worthy finding a better way to determine $n_{\max }$ instead of setting an arbitrary large number. For one thing, extra computation complexities are introduced if the model order is too large. For another, spurious modes including physical modes and mathematical modes appear when model order is unreasonably selected.

This paper proposes a model order selection method based on singular entropy theory. The concept of entropy was first proposed by a German physicist Crawhuse in 1865. Entropy is an index to quantify the uncertainty of a system under a specific state. Suppose a system has $m$ states, the probability of each state is $p_{i}(i=1,2, \ldots, m)$, and then the entropy of this system $E$ is defined as

$$
E=-\sum_{i=1}^{m} p_{i} \ln p_{i}
$$

Follow the same rule; the singular entropy can be introduced. Zhang [28] first introduced singular entropy into the field of modal analysis for model order selection. However, the singular entropy is calculated from principal angles in Zhang's method, which is only suitable for data-driven SSI. When applying SVD to the Toeplitz matrix, a sequence of eigenvalues of Toeplitz matrix are obtained. Under the condition of noiseless or high signal noise ratio, the eigenvalues matrix $\Lambda$ of output covariance matrix should contain zero values, described by

$$
\Lambda=\operatorname{diag}\left(\lambda_{1}, \lambda_{2}, \ldots, \lambda_{l}, 0,0, \ldots, 0\right),
$$

where $\lambda_{1} \geq \lambda_{2} \geq \cdots \geq \lambda_{l}>0$. Theoretically, the model order equals $l$. While in practice, the output covariance calculated from output measurements is influenced by noise. The eigenvalues of output covariance matrix converge to zeros with the increase of model order. Thus, $\Lambda$ is described as

$$
\Lambda=\operatorname{diag}\left(\lambda_{1}, \lambda_{2}, \ldots, \lambda_{m}\right),
$$

where $\lambda_{1} \geq \lambda_{2} \geq \cdots \geq \lambda_{m}>0$. From (12) and (13), it is obvious that the number of nonzero eigenvalues shows the level of output measurements influenced by noise. The less number of nonzero eigenvalues in $\Lambda$, the output measurements are less influenced by noise, meaning that a higher signal noise ratio is expected. The eigenvalue matrix $\Lambda$ reflects the information of output measurements. Therefore, the singular entropy $E_{k}$ can be similarly defined as

$$
E_{k}=\sum_{i=1}^{k} \Delta E_{i}
$$

where $k$ is the order of singular entropy and $\Delta E_{i}$ is the increment of the singular entropy at order $i$, obtained from

$$
\Delta E_{i}=-\left(\frac{\lambda_{i}}{\sum_{i=1}^{n} \lambda_{i}}\right) \ln \left(\frac{\lambda_{i}}{\sum_{i=1}^{n} \lambda_{i}}\right) .
$$

Equation (15) has the same form with (11). Considering the property of eigenvalues, the singular entropy increment $\Delta E_{i}$ has high value at lower model orders, and it approaches to a fixed value from a specific model order. Under this specific model order, the effective information of output covariance 
matrix can be extracted. The first order variation of $\Delta E_{i}$ can be used to determine the specific model order. The model order corresponding to the variation of $\Delta E_{i}$ which approaches to 0 can be selected as the model order as shown in the simulation example and real word application.

2.3. SD with Confidence Intervals. Although the original intention of introducing SD is to solve the problem of spurious mode, while in practice, spurious mode is still a main issue in postprocessing of modal analysis. This issue becomes even worse when the model order is unreasonably chosen (too big). In addition, the ambient vibration test of civil engineering structures is often divided into several setups, meaning that several SDs need to be analyzed. This could be a time-consuming process, and sometimes the spurious mode is mistaken for system mode. If an index can be introduced in $\mathrm{SD}$, so that the credibility of modal parameters at each model order is directly shown; then the spurious mode issue can be greatly restrained. This paper introduces the uncertainty of modal parameters as the index to distinguishing system mode from spurious mode. The confidence interval can be constructed from the uncertainty of modal parameters. Usually, the system modes have lower uncertainties (small confidence intervals), while the spurious modes have higher uncertainties (large confidence intervals). Through setting a threshold to uncertainty of modal parameters, the spurious modes can be automatically removed from SD.

The reasons that lead to the uncertainty of experimentally identified modal parameters include the following: (1) the noises during vibration test; (2) the finite data samples; (3) the inaccuracies in modelling and computation. The uncertainty of modal parameters extracted from SSI-cov can be quantified by sensitive analysis of system matrices. From (3), it is known that the output covariance converges to its true value when data samples go to infinite. While in a real test, the length of data samples is finite. Then, the calculated output covariance is only an estimate $\widehat{R}_{i}$ of its true value, expressed by

$$
\widehat{R}_{i}=\frac{1}{N} \sum_{k=0}^{N-i} y_{k+i} y_{k}^{T}
$$

Since the system matrices $(A, C)$ are obtained by decomposing output covariance matrix, the extracted system matrices are also only an estimate $(\widehat{A}, \widehat{C})$ of their true values. Then, it follows that

$$
\operatorname{cov}\left(f_{i}, \xi_{i}, \phi_{i}\right)=\zeta_{f_{i}, \xi_{i}, \phi_{i}} \Sigma_{(\widehat{A}, \widehat{C})} \zeta_{f_{i}, \xi_{i}, \phi_{i}}^{T}
$$

where $\operatorname{cov}\left(f_{i}, \xi_{i}, \phi_{i}\right)$ is the uncertainty of modal parameters; $\zeta_{f_{i}, \xi_{i}, \phi_{i}}$ is the sensitivity of modal parameters to the first perturbation of system matrices; $\Sigma_{(\widehat{A}, \widehat{C})}$ is the covariance of the system matrices. In [22], the detailed mathematical formulation of uncertainty quantification is provided.

\section{Simulation Example}

To test the effectiveness of the proposed model order selection method and illustrate the practicability of SD with confidence

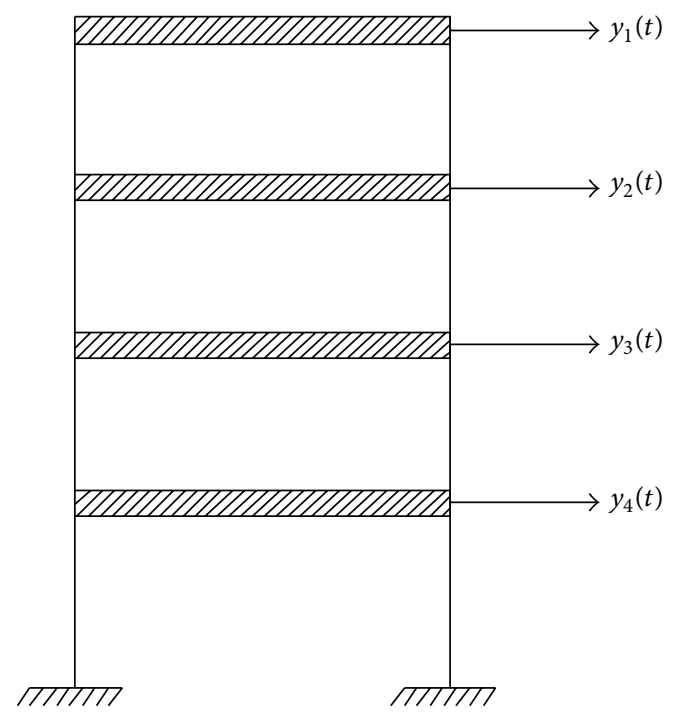

FIgURE 1: The four-story structure.

intervals, a simulation of a four-story structure is studied in this section. The four-story structure (Figure 1) has mass matrix $M$, stiffness matrix $K$, and damping matrix $C_{v}$ as follows:

$$
\begin{aligned}
& M=\left[\begin{array}{llll}
3 & 0 & 0 & 0 \\
0 & 3 & 0 & 0 \\
0 & 0 & 3 & 0 \\
0 & 0 & 0 & 3
\end{array}\right] \mathrm{kg} \\
& K=4000\left[\begin{array}{cccc}
2 & -1 & \\
-1 & 2 & -1 & \\
-1 & 2 & -1 \\
& & -1 & 2
\end{array}\right] \mathrm{N} / \mathrm{m} ; \\
& C_{v}=\frac{K}{1000} \mathrm{~N} /(\mathrm{m} / \mathrm{s}) .
\end{aligned}
$$
follows:

The theoretical solution of the modal parameters is as

$$
\begin{aligned}
& f=(2.018,5.812,8.904,10.922) \mathrm{Hz} ; \\
& \xi=(0.63,1.83,2.80,3.43) \% .
\end{aligned}
$$

The simulation process mainly includes three steps: exert excitations modelled by white noise; record the accelerations of the frame structure; extract modal parameters by SSIcov algorithm. At the third step, the proposed model order selection method and SD with confidence intervals can be verified.

3.1. Verification of Model Order Selection Method. To determine the model order, the singular value decomposition is applied to the output covariance matrix obtained from output vector of the simulation. The singular value versus model order plot is shown in Figure 2. By observing Figure 2, one 


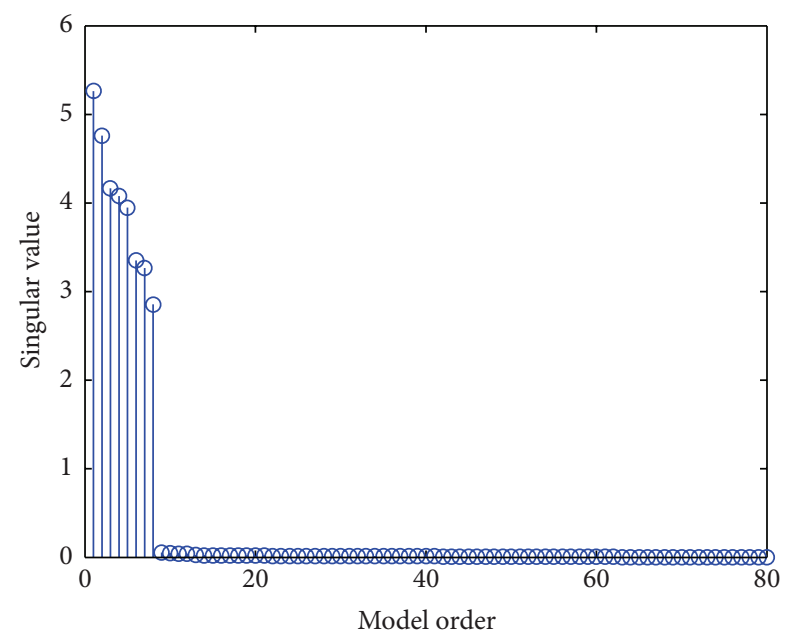

FIGURE 2: The frame structure: singular values of the output covariance matrix.

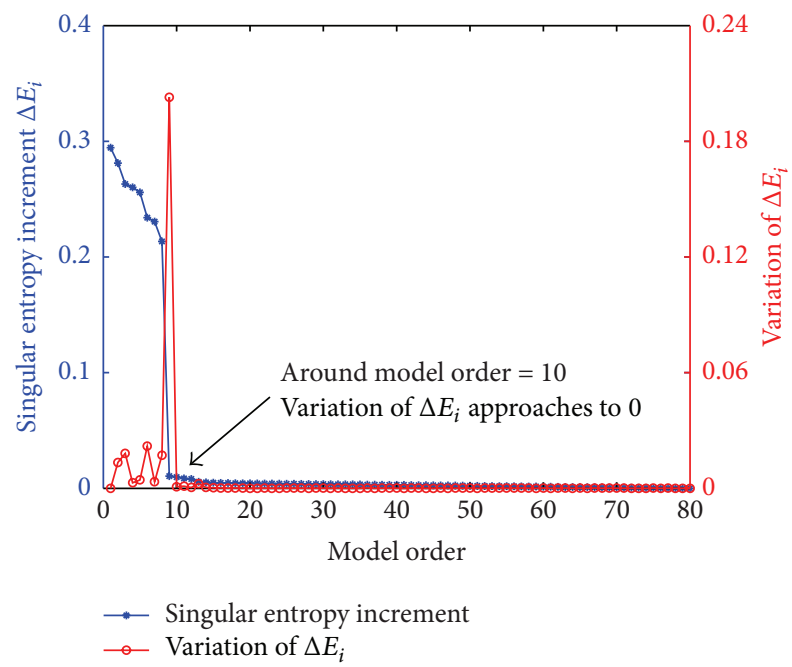

FIGURE 3: The frame structure: the change of singular entropy increment and its first-order variation to model orders.

can see that 4 pairs of nonzero singular values exist, meaning that the true model order of the frame structure is 8 . In real engineering application, the change of singular values with model orders is not so obvious. This brings difficulties for model order selection. According to (15), the singular entropy increment $\Delta E_{i}$ at each model order can be calculated. The first-order variation of $\Delta E_{i}$ can be easily obtained. Figure 3 shows the change of $\Delta E_{i}$ and its first-order variation with model order. Starting from model order 10, both singular entropy increment and its variation converge to zero. Thus, the model order is selected as 10 , which is very close to its true value. The simulation demonstrates that based on singular entropy theory the model order can be reasonably selected.

3.2. Verification of SD with Confidence Intervals. To illustrate the effectiveness of SD with confidence intervals in dealing with spurious modes, the model order is selected as 20 so that mathematical modes show up. Computing the uncertainties of modal parameters at each model order and the frequency with confidence intervals $\left(f \pm \sigma_{f}\right)$ versus model order plot is shown in Figure 4(a). In Figure 4(a), the circles stand for the stable points and the error bar is the confidence intervals. The simulated four-story structure theoretically has four modes, while one expects five obvious stable poles in Figure 4(a). This means that when model order is set too big, some stable points representing spurious mode are gradually forming new stable poles. The users need to distinguish which pole is system mode. By setting a threshold for frequency uncertainty, making that the SD presents stable points with frequency uncertainty smaller than 0.1 , a much clear SD can be obtained. In Figure 4(b), the stable pole around $13 \mathrm{~Hz}$ is automatically removed; only four system modes can be found. This kind of "clear" SD will remarkably reduce GUI time, providing more robust results.

\section{Application: OMA of Ligong Footbridge}

4.1. Bridge Description. The Ligong footbridge, located at Wuhan, is a single pylon steel cable-stayed footbridge. The total length of the footbridge is $116.7 \mathrm{~m}$ with a main span of $45 \mathrm{~m}$. The steel pylon is as high as $24.5 \mathrm{~m}$. At each side of the pylon, four parallel stay-cables are anchored at the center of the bridge deck. The width of bridge deck is $7.0 \mathrm{~m}$. The main girder is composed of two parts: the straight line part and $\mathrm{U}$ arc part. The elevation and plan view of the footbridge are shown in Figure 5.

4.2. Ambient Vibration Test. To evaluate the dynamic property and also gather useful data for serviceability assessment of the footbridge, an ambient vibration test campaign was carried out. The ambient vibration test makes use of the operational response to freely accessible ambient loads (wind, traffic, pedestrian, microtremors, etc.). The lower modes of large civil structure can be fully excited under ambient loads. During an ambient vibration test, the structure under test remains in operation. Thus, ambient vibration test has become very popular for OMA of civil structures. The test campaign for Ligong footbridge was based on 5 wireless acceleration sensors, two of which are fixed as reference sensors and the left are roving sensors. In total, the biaxial acceleration responses of 53 test points on the bridge deck divided into 17 setups were recorded. Thus, each setup has data of four reference channels and six roving channels. The sampling frequency was $200 \mathrm{~Hz}$, and the sampling time for each setup was around 15 minutes. Figure 6 shows the measurement grid of the footbridge deck.

4.3. Operational Modal Analysis. Before operational modal analysis of the footbridge is performed, the following preprocessing operations of the output responses are accomplished:

(1) The constant offset is removed.

(2) The low frequency noise is removed by high-pass filter with a cut-off frequency $0.1 \mathrm{~Hz}$.

(3) Resample the data from $200 \mathrm{~Hz}$ to $67 \mathrm{~Hz}$. 


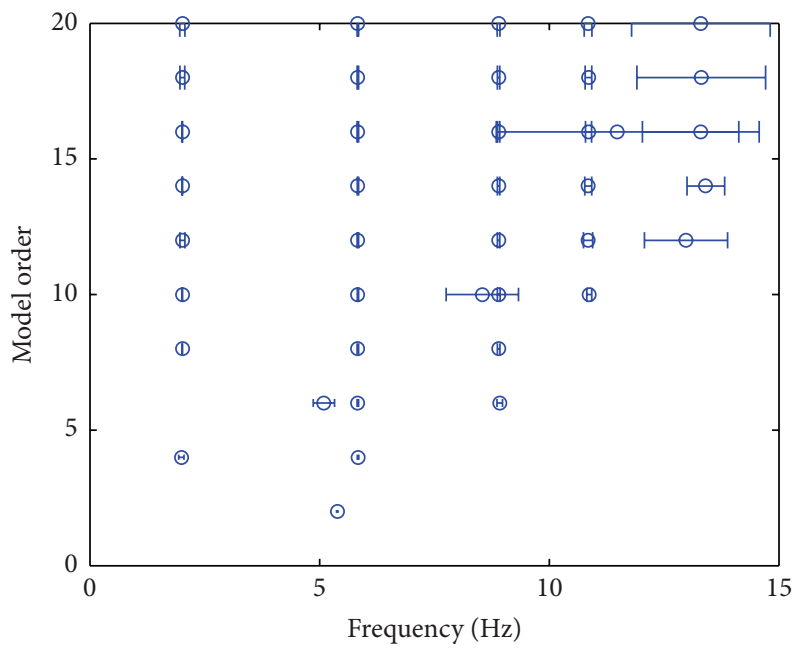

(a) All stable points

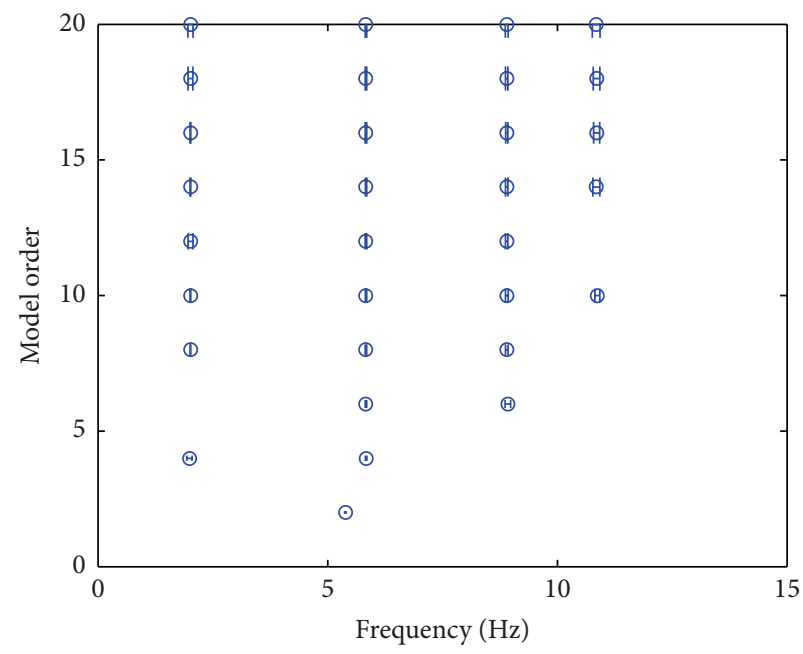

(b) Stable points with $\sigma_{f} \leq 0.1$

FIGURE 4: Stabilization diagram with confidence intervals of the frame structure.
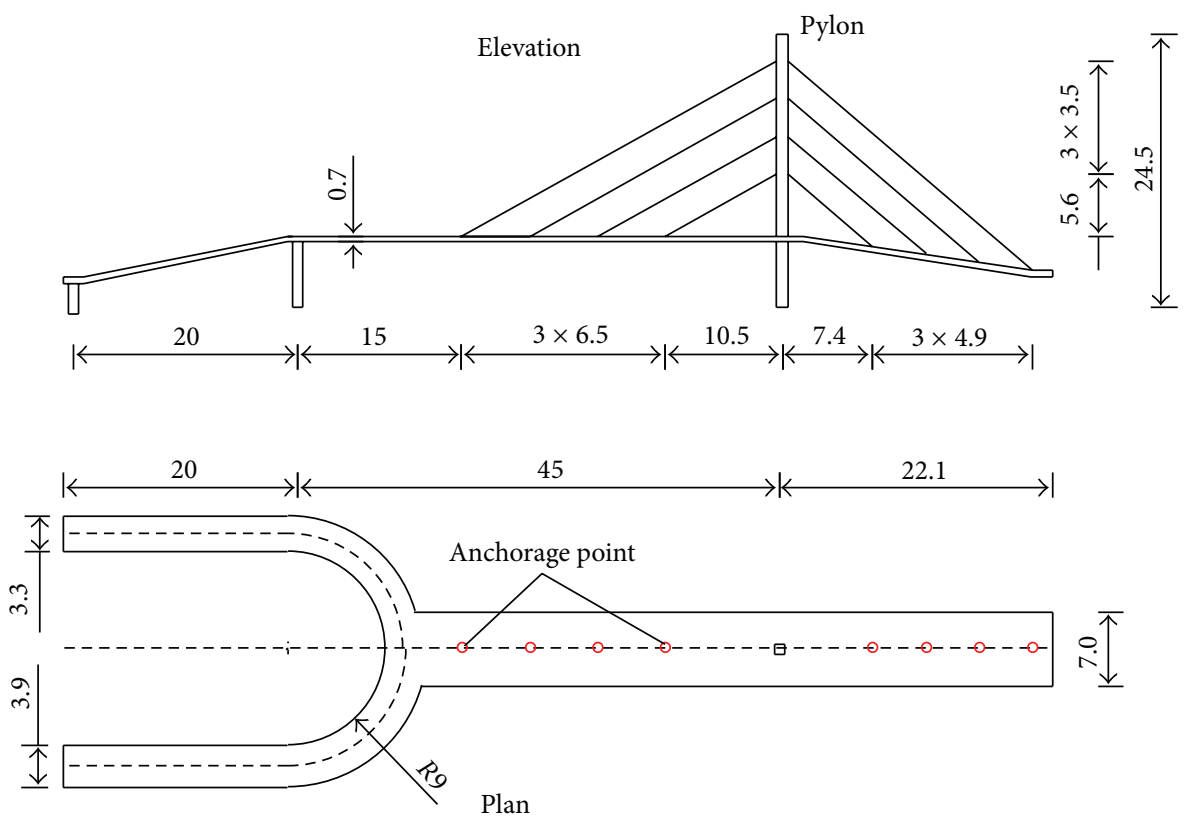

FIGURE 5: The elevation and plan of the footbridge (dimensions are in $\mathrm{m}$ ).

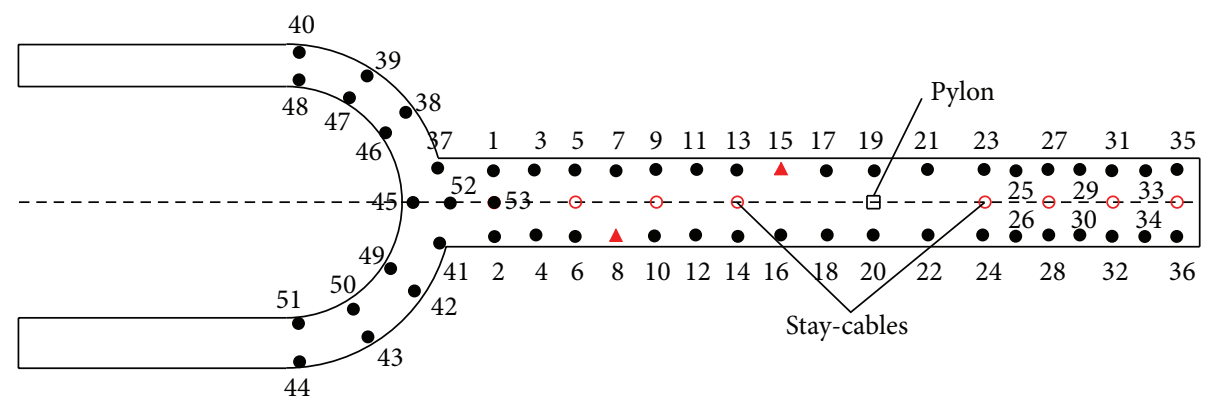

FIGURE 6: The footbridge: measurement grid, node numbers, and reference locations. (The triangles represent reference sensors and the circles indicate roving sensors.) 


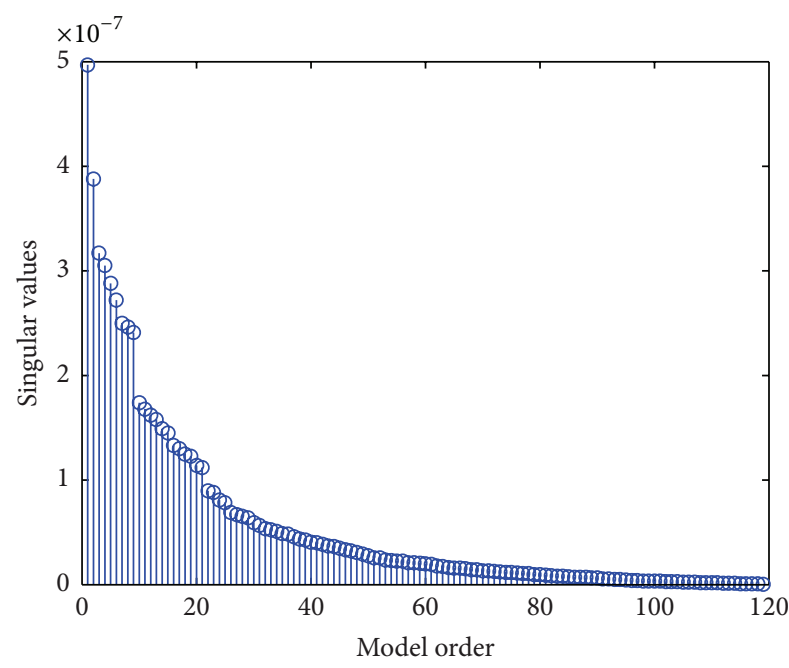

Figure 7: The footbridge: the singular values of output covariance matrix.

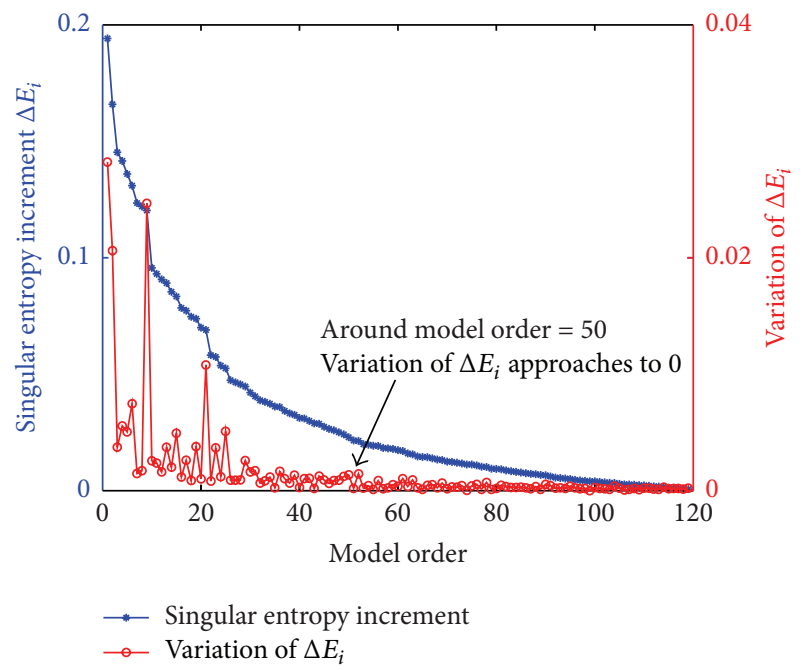

FIGURE 8: The footbridge: the change of singular entropy increment and its variation with model order.

4.4. Model Order Selection by Singular Entropy. To determine model order, the singular values of output covariance matrix are shown in Figure 7. Duo to the influence of noise, the singular values do not have a big "jump" at specific model order from nonzero value to zero as shown in the simulation example. The singular values in Figure 7 gradually converge to zero with the increase of model order. Thus, it is difficult to determine model order by observing a singular value versus model order plot. Figure 8 shows the singular entropy increment and its variation change with model order. Starting from model order equal to 50 (approximately), the variation of singular entropy increment stably approaches to 0 . According to the method proposed in this paper, the model order should be selected as 50. Compared to the simulation example, the singular values and singular entropy increment change with model order show no big jump but only converge to zero with the increase of model order. However, the variation of
TABLE 1: The analytically and experimentally determined natural frequencies and damping ratios.

\begin{tabular}{lccccc}
\hline \multirow{2}{*}{ Mode } & FEA & PP & \multicolumn{2}{c}{ SSI-cov } & \multirow{2}{*}{ MPC } \\
& $f[\mathrm{~Hz}]$ & $f[\mathrm{~Hz}]$ & $f[\mathrm{~Hz}]$ & $\xi[\%]$ & \\
\hline First vertical bending & 2.16 & 2.08 & 2.10 & 0.594 & 0.876 \\
First torsion & 4.68 & 4.59 & 4.52 & 0.673 & 0.891 \\
Second vertical bending & 6.11 & 5.88 & 5.90 & 0.645 & 0.856 \\
Third vertical bending & 9.40 & 9.34 & 9.29 & 1.032 & 0.835 \\
\hline
\end{tabular}

singular entropy increment changes with model order in the full-scale application follows the same principle with that in the mathematical simulation.

4.5. SD with Confidence Intervals. Set the model order as 50 and then compute the modal parameters and corresponding uncertainties at each order. Figure 9(a) is the SD with frequency confidence intervals obtained from setup 1 . In Figure 9(a), excepting four obvious stable poles, it can be seen that some stable points scatter over the plot and the stable poles gradually formed. One needs to check whether these stable poles stand for system mode. By setting threshold for frequency uncertainty, a much clear SD is obtained, as shown in Figure 9(b). The spurious modes issue has been greatly restrained in SD with confidence intervals.

In total, 4 lower modes, including 3 vertical bending modes and 1 torsion mode, are extracted from operational modal analysis. Table 1 shows the analytically and experimentally determined modal parameters. The analytical results are obtained from finite element analysis (FEA, see Figure 10). The results from and Peak-Picking are also listed for comparison. As it can be seen from Table 1, the frequencies from FEA are usually a little bigger than that from the experiment, while a good agreement is achieved between PP and SS-cov. This is because the FE model is established from design documents, while the physical structure may be damaged. Therefore, the FE model needs further updating and calibration. As for the damping ratios, the experimental results show no good harmony and distribution. This is because the damping mechanism of large civil structures is complicated; thus the error bounds for damping ratio estimates could be large (see, for instance, [19]). Figure 11 shows the corresponding mode shapes of the four extracted modes. The comparatively high modal phase collinearity (MPC) in Table 1 shows that the mode shapes are well identified from SSI-cov.

\section{Conclusions}

The stabilization diagram has become a standard tool in many time-domain modal identification algorithms. In this paper, an improved stabilization diagram for subspace-based algorithms has been proposed to make it better applied in stochastic subspace identification. Based on singular entropy theory, a model order selection method has been proposed. Besides, stabilization diagram with confidence intervals constructed from quantified uncertainties of modal parameters has been presented. The improved stabilization diagram has been illustrated by both the simulation example of a four-story 


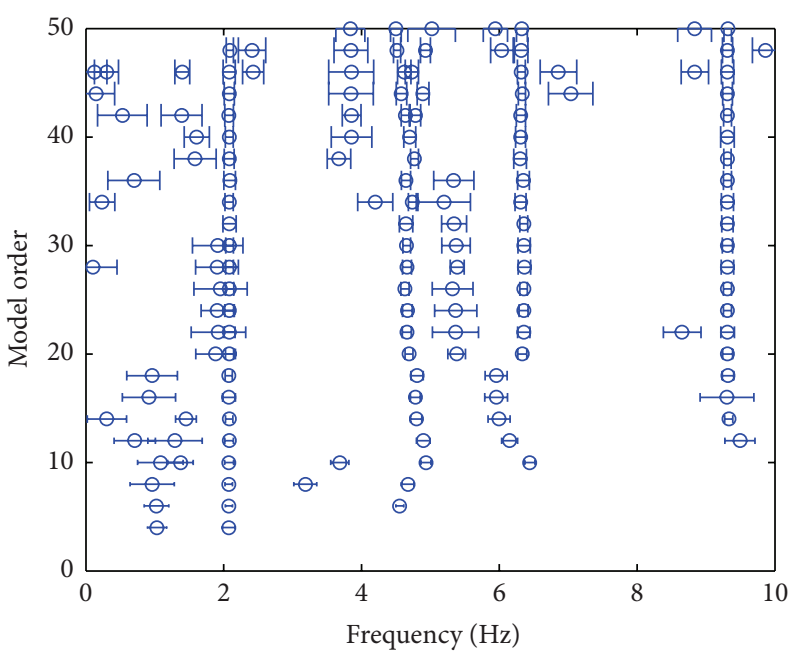

(a) All stable points

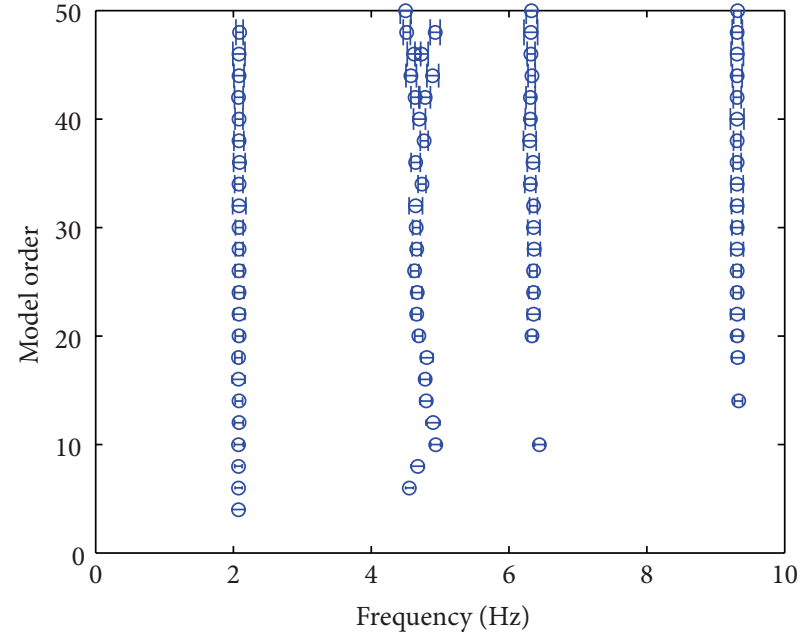

(b) Stable points with $\sigma_{f} \leq 0.1$

FIgURE 9: The footbridge: SD with confidence.

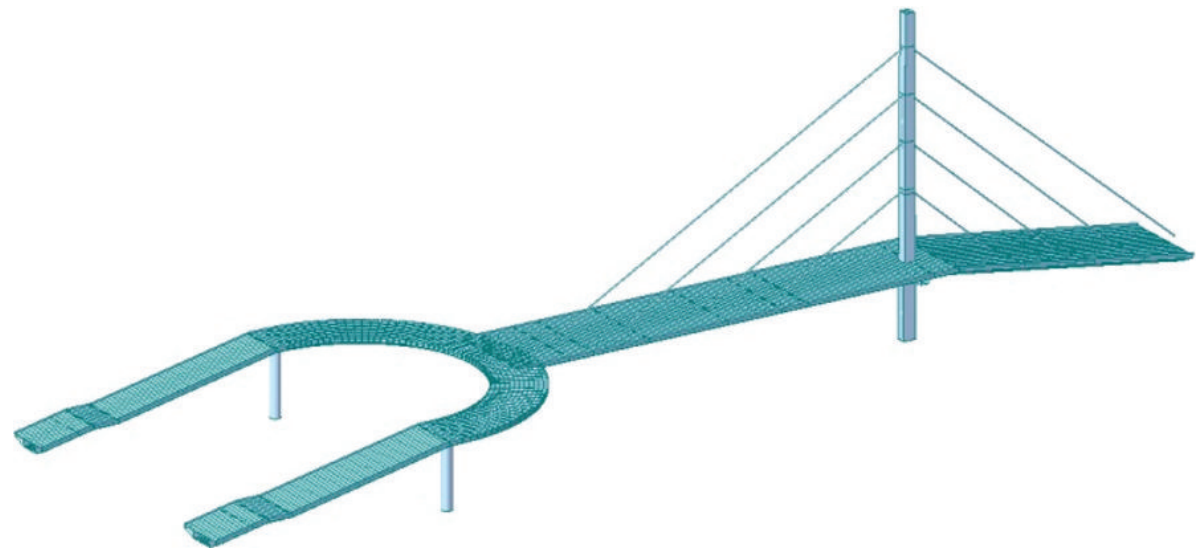

FIgURE 10: The finite element model of Ligong footbridge.

structure and the application of Ligong footbridge. Conclusions can be made as follows:

(1) The model order selection method is based on the calculation of singular entropy increment and its first-order variation. Start from model order $n$; the variation of singular entropy increment approaches to zero; then $n$ can be determined as model order. The proposed method is easy to be implemented, avoiding the computation complexities and spurious modes introduced by unreasonable selected model order. As can be seen from the proposed method, the model order selection essentially relies on the singular value decomposition of the block Toeplitz matrix. By introducing the variation of singular entropy increment, the noise effects that make singular values all different from 0 have been eliminated.

(2) Stabilization diagram with confidence intervals is an effective tool to distinguish system modes and spurious modes. By setting a threshold for the confidence intervals of stable points, the spurious modes can be automatically removed, making a much clear stabilization diagram. This kind of clear stabilization diagram can reduce the time spent on postprocessing of modal analysis and provide more accurate results.

It is worth mentioning that the improved stabilization diagram method is generally applicable and not dependent on the structure type.

\section{Conflict of Interests}

The authors declare that there is no conflict of interests regarding the publication of this paper.

\section{Acknowledgments}

This work was supported by the Natural Science Foundation of Hubei Province, Grant no. 2015CFB393, and the Fundamental Research Foundation of Central Universities, Grant 


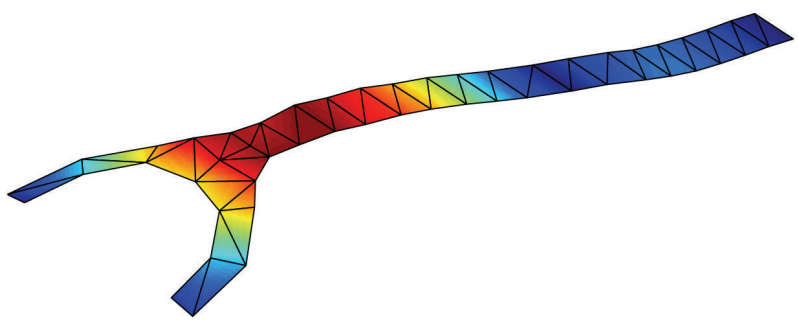

Mode 1: vertical bending, $f=2.10 \mathrm{~Hz}$

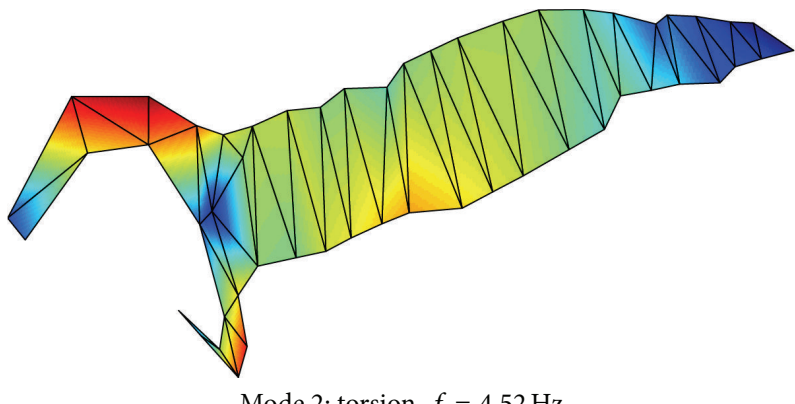

Mode 2: torsion, $f=4.52 \mathrm{~Hz}$

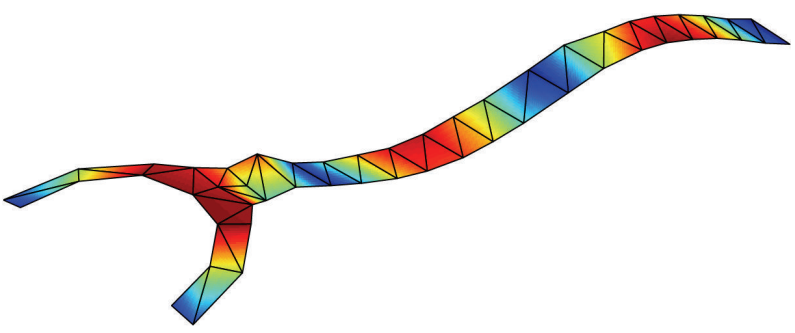

Mode 3: vertical bending, $f=5.90 \mathrm{~Hz}$

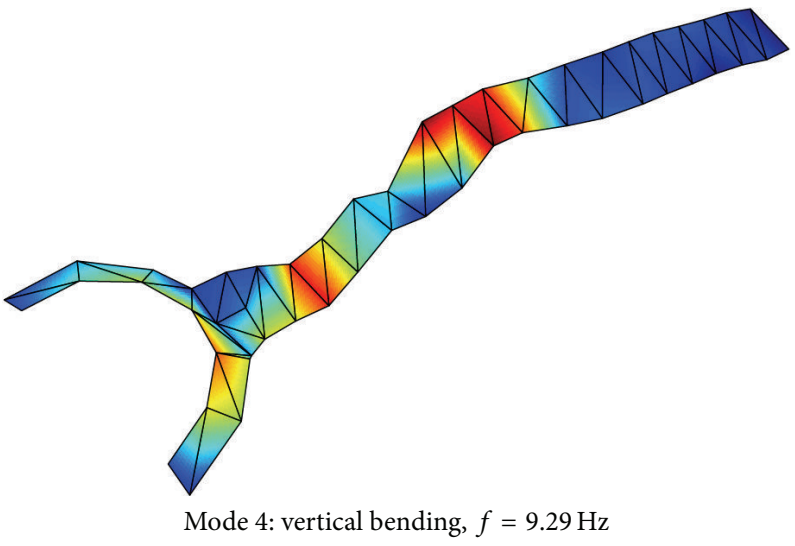

FIGURE 11: The footbridge: vertical bending and torsion mode shapes.

no. WUT-2014-IV-047. The modal parameters of Ligong footbridge were extracted based on MACEC 3.2, a Matlab toolbox for modal analysis developed by Professor Edwin Reynders and Guido De Roeck.

\section{References}

[1] C. R. Farrar, T. Duffey, J. P. Cornwell et al., "Excitation methods for bridge structures," in Proceedings of the 17th International Modal Analysis Conference, pp. 1063-1068, Society for Experimental Mechanics, Kissimmee, Fla, USA, February 1999.
[2] C. Rainieri and G. Fabbrocino, Operational Modal Analysis of Civil Engineering Structures, Springer, New York, NY, USA, 2014.

[3] E. Reynders, A. Teughels, and G. De Roeck, "Finite element model updating and structural damage identification using OMAX data," Mechanical Systems and Signal Processing, vol. 24, no. 5, pp. 1306-1323, 2010.

[4] B. Jaishi, H.-J. Kim, M. K. Kim, W.-X. Ren, and S.-H. Lee, "Finite element model updating of concrete-filled steel tubular arch bridge under operational condition using modal flexibility," Mechanical Systems and Signal Processing, vol. 21, no. 6, pp. 2406-2426, 2007. 
[5] M. I. Friswell and J. E. Mottershead, Finite Element Model Updating in Structural Dynamics, Kluwer Academic, Dordrecht, The Netherlands, 1995.

[6] G. De Roeck, "The state-of-the-art of damage detection by vibration monitoring: the SIMCES experience," Journal of Structural Control, vol. 10, no. 2, pp. 127-134, 2003.

[7] M. M. A. Wahab and G. De Roeck, "Damage detection in bridges using modal curvatures: application to a real damage scenario," Journal of Sound and Vibration, vol. 226, no. 2, pp. 217-235, 1999.

[8] Y. Xia, H. Hao, J. M. W. Brownjohn, and P.-Q. Xia, "Damage identification of structures with uncertain frequency and mode shape data," Earthquake Engineering and Structural Dynamics, vol. 31, no. 5, pp. 1053-1066, 2002.

[9] C. Gentile and A. Saisi, "Ambient vibration testing and condition assessment of the Paderno iron arch bridge (1889)," Construction and Building Materials, vol. 25, no. 9, pp. 3709$3720,2011$.

[10] C. Gentile and N. Gallino, "Condition assessment and dynamic system identification of a historic suspension footbridge," Structural Control and Health Monitoring, vol. 15, no. 3, pp. 369-388, 2008.

[11] C. Rainieri, G. Fabbrocino, G. Manfredi, and M. Dolce, "Robust output-only modal identification and monitoring of buildings in the presence of dynamic interactions for rapid postearthquake emergency management," Engineering Structures, vol. 34, pp. 436-446, 2012.

[12] C. Rainieri, G. Fabbrocino, and F. S. De Magistris, "An integrated seismic monitoring system for a full-scale embedded retaining wall," Geotechnical Testing Journal, vol. 36, no. 1, pp. 1-14, 2013.

[13] C. Rainieri, G. Fabbrocino, and E. Cosenza, "Integrated seismic early warning and structural health monitoring of critical civil infrastructures in seismically prone areas," Structural Health Monitoring, vol. 10, no. 3, pp. 291-308, 2011.

[14] Y.-C. Liu, C.-H. Loh, and Y.-Q. Ni, "Stochastic subspace identification for output-only modal analysis: application to super high-rise tower under abnormal loading condition," Earthquake Engineering and Structural Dynamics, vol. 42, no. 4, pp. 477498, 2013.

[15] B. Peeters and G. De Roeck, "Stochastic system identification for operational modal analysis: a review," Journal of Dynamic Systems, Measurement, and Control, vol. 123, no. 4, pp. 659-667, 2001.

[16] D.-J. Yu and W.-X. Ren, "EMD-based stochastic subspace identification of structures from operational vibration measurements," Engineering Structures, vol. 27, no. 12, pp. 1741-1751, 2005.

[17] A. C. Altunişik, A. Bayraktar, B. Sevim, and H. Özdemir, "Experimental and analytical system identification of Eynel arch type steel highway bridge," Journal of Constructional Steel Research, vol. 67, no. 12, pp. 1912-1921, 2011.

[18] P. V. Overschee, B. De Moor, D. A. Hensher et al., Subspace Identification for the Linear Systems: Theory-Implementation, Kluwer Academic Publishers, 1996.

[19] C. Rainieri, G. Fabbrocino, and E. Cosenza, "Some remarks on experimental estimation of damping for seismic design of civil constructions," Shock and Vibration, vol. 17, no. 4-5, pp.383-395, 2010.
[20] E. Reynders, R. Pintelon, and G. De Roeck, "Uncertainty bounds on modal parameters obtained from stochastic subspace identification," Mechanical Systems and Signal Processing, vol. 22, no. 4, pp. 948-969, 2008.

[21] M. Döhler, X.-B. Lam, and L. Mevel, "Uncertainty quantification for modal parameters from stochastic subspace identification on multi-setup measurements," Mechanical Systems and Signal Processing, vol. 36, no. 2, pp. 562-581, 2013.

[22] C. Rainieri, "Perspectives of second-order blind identification for operational modal analysis of civil structures," Shock and Vibration, vol. 2014, Article ID 845106, 9 pages, 2014.

[23] C. Geng, F. Wang, J. Zhang, and Z. Jin, "Modal parameters identification of power transformer winding based on improved empirical mode decomposition method," Electric Power Systems Research, vol. 108, pp. 331-339, 2014.

[24] G. Zhang, J. Ma, Z. Chen, and R. Wang, "Automated eigensystem realisation algorithm for operational modal analysis," Journal of Sound and Vibration, vol. 333, no. 15, pp. 3550-3563, 2014.

[25] F. Ubertini, C. Gentile, and A. L. Materazzi, "Automated modal identification in operational conditions and its application to bridges," Engineering Structures, vol. 46, pp. 264-278, 2013.

[26] C. Rainieri and G. Fabbrocino, "Automated output-only dynamic identification of civil engineering structures," Mechanical Systems and Signal Processing, vol. 24, no. 3, pp. 678-695, 2010.

[27] C. Rainieri and G. Fabbrocino, "Influence of model order and number of block rows on accuracy and precision of modal parameter estimates in stochastic subspace identification," International Journal of Lifecycle Performance Engineering, vol. 1, no. 4, pp. 317-334, 2014.

[28] X. Zhang, Stochastic subspace identification and application of structure under ambient vibration [M.S. thesis], Fuzhou University, Fuzhou, China, 2006. 


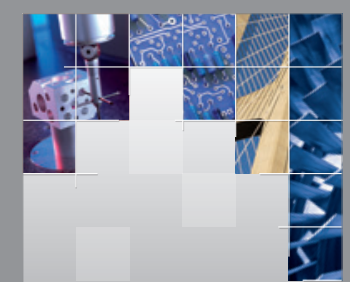

\section{Enfincering}
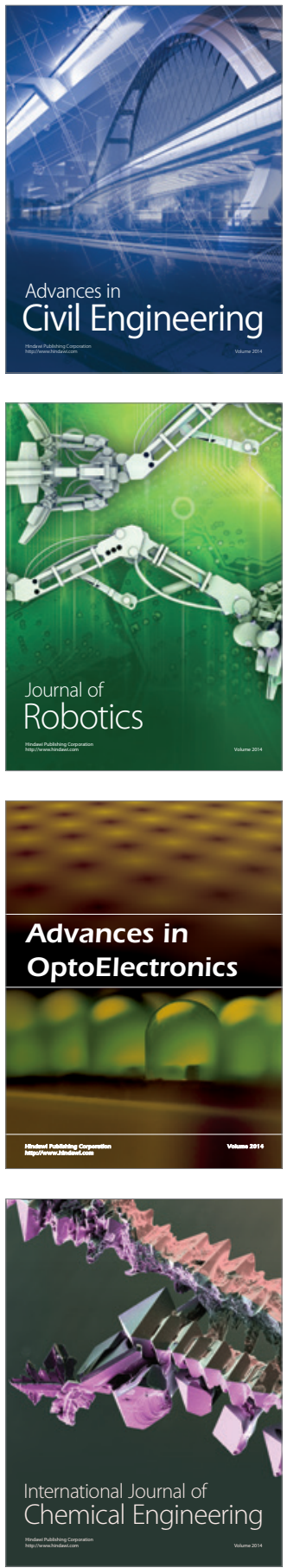

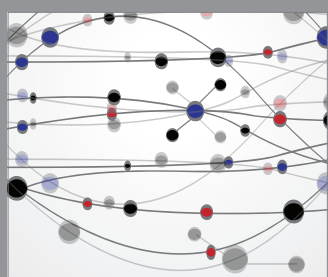

The Scientific World Journal

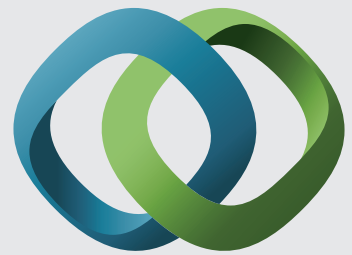

\section{Hindawi}

Submit your manuscripts at

http://www.hindawi.com
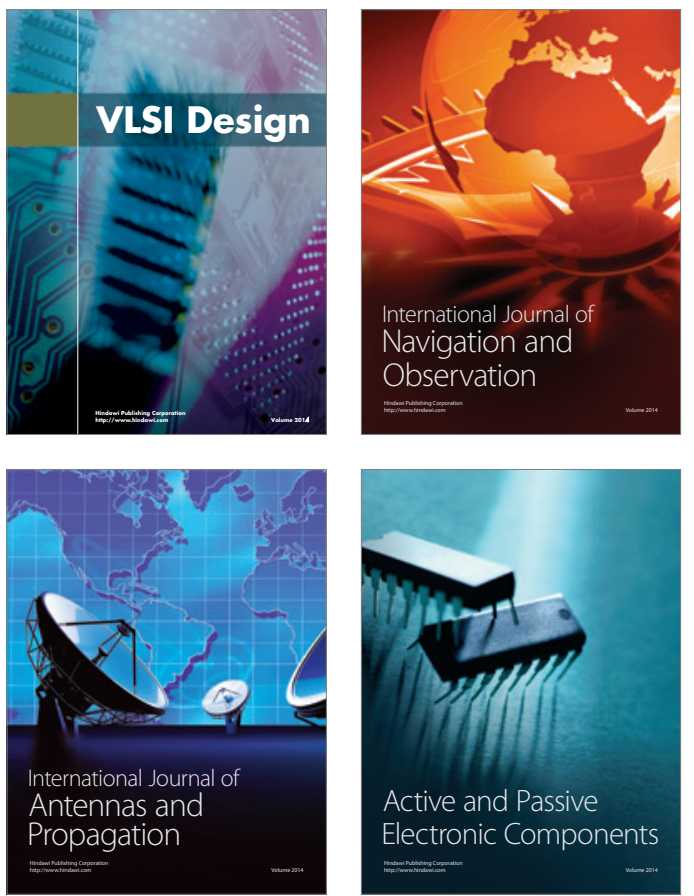
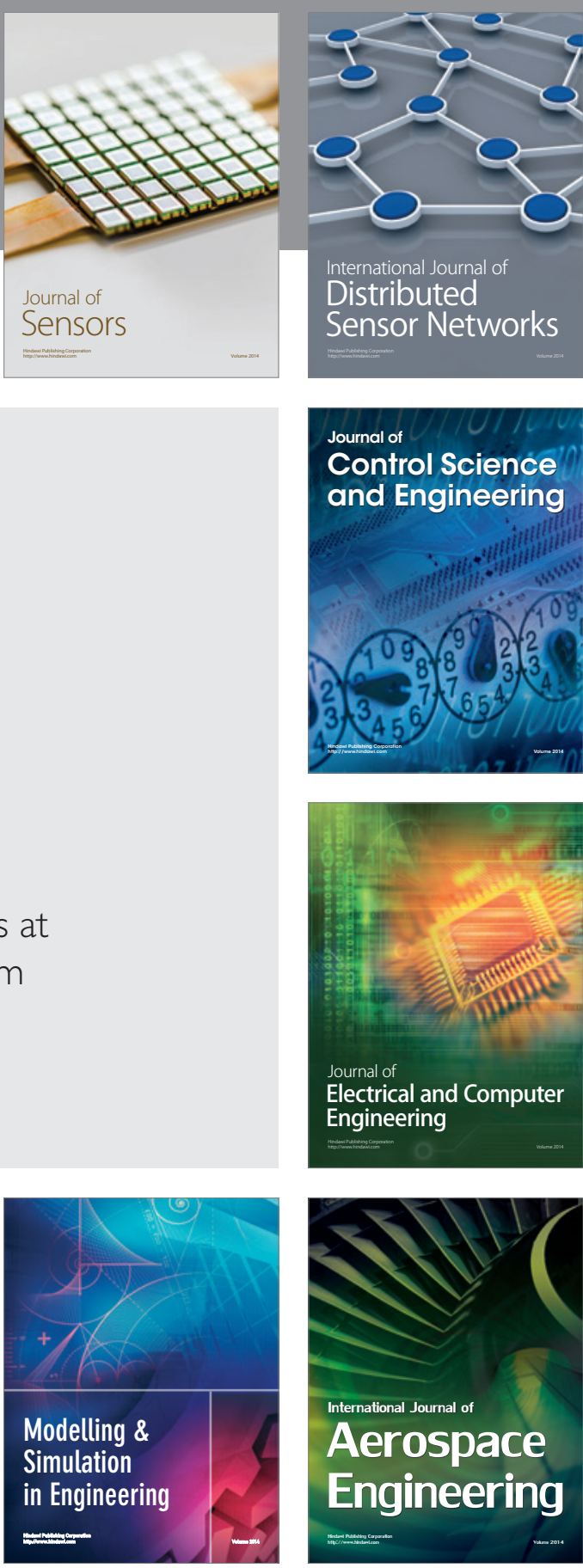

International Journal of

Distributed

Sensor Networks

Journal of

Control Science

and Engineering
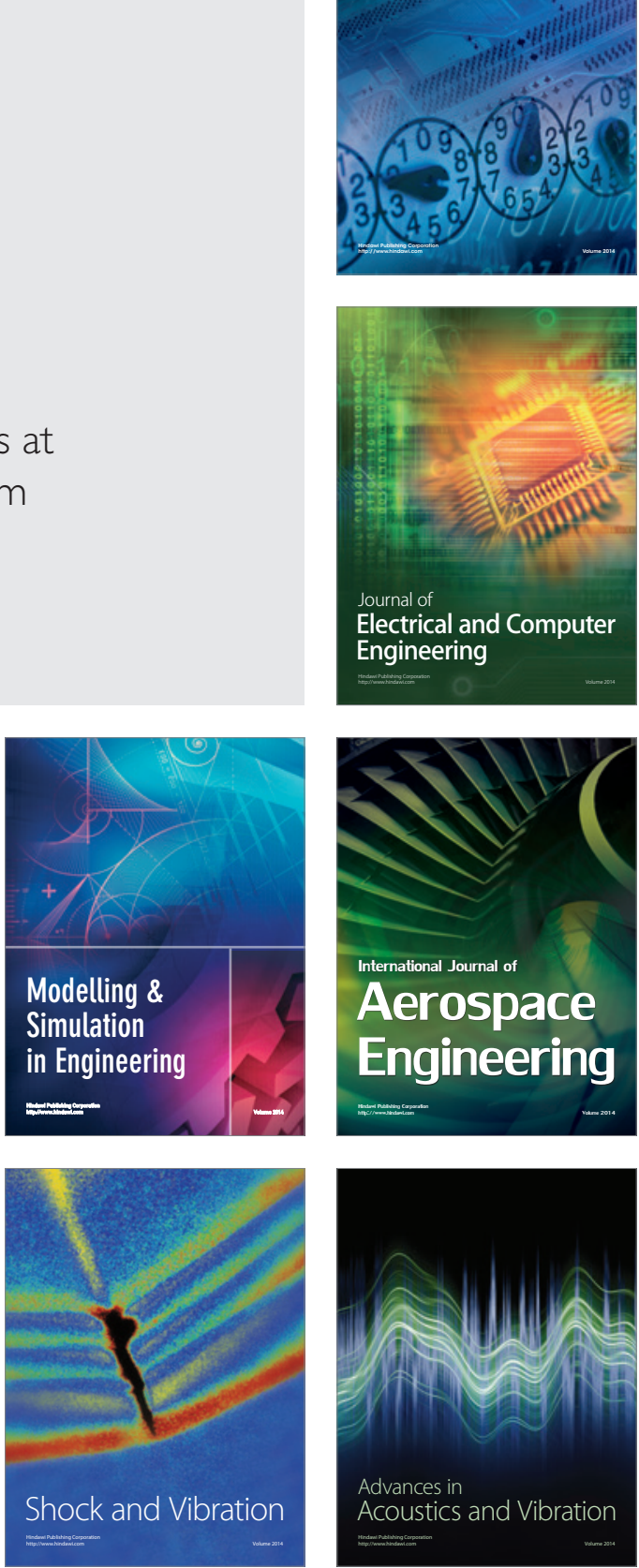